\title{
Localising occult prostate cancer metastasis with advanced imaging techniques (LOCATE trial): a prospective cohort, observational diagnostic accuracy trial investigating whole-body magnetic resonance imaging in radio-recurrent prostate cancer
}

Sola Adeleke ${ }^{1 \dagger}$, Arash Latifoltojar $^{1 \dagger}$, Harbir Sidhu ${ }^{1,2}$, Myria Galazi $^{3}$, Taimur T. Shah ${ }^{4,5,6}$, Joey Clemente ${ }^{1}$, Reena Davda ${ }^{7}$, Heather Ann Payne ${ }^{7}$, Manil D. Chouhan ${ }^{1,2}$, Maria Lioumi ${ }^{8}$, Sue Chua ${ }^{9}$, Alex Freeman $^{10}$, Manuel Rodriguez-Justo ${ }^{10}$, Anthony Coolen ${ }^{11}$, Sachin Vadgama ${ }^{12}$, Steve Morris ${ }^{12}$, Gary J. Cook ${ }^{13}$, Jamshed Bomanji ${ }^{14}$, Manit Arya ${ }^{15}$, Simon Chowdhury ${ }^{16}$, Simon Wan ${ }^{14}$, Athar Haroon ${ }^{17}$, Tony Ng ${ }^{3}$, Hashim Uddin Ahmed ${ }^{5,18}$ and Shonit Punwani ${ }^{1,2^{*}}$

\footnotetext{
Abstract

Background: Accurate whole-body staging following biochemical relapse in prostate cancer is vital in determining the optimum disease management. Current imaging guidelines recommend various imaging platforms such as computed tomography (CT), Technetium $99 \mathrm{~m}\left({ }^{99 \mathrm{~m}} \mathrm{Tc}\right)$ bone scan and ${ }^{18} \mathrm{~F}$-choline and recently ${ }^{68} \mathrm{Ga}-\mathrm{PSMA}$ positron emission tomography (PET) for the evaluation of the extent of disease. Such approach requires multiple hospital attendances and can be time and resource intensive. Recently, whole-body magnetic resonance imaging (WB-MRI) has been used in a single visit scanning session for several malignancies, including prostate cancer, with promising results, providing similar accuracy compared to the combined conventional imaging techniques. The LOCATE trial aims to investigate the application of WB-MRI for re-staging of patients with biochemical relapse (BCR) following external beam radiotherapy and brachytherapy in patients with prostate cancer.

(Continued on next page)
}

\footnotetext{
* Correspondence: shonit.punwani@gmail.com

†'Sola Adeleke and Arash Latifoltojar contributed equally to this work.

${ }^{1}$ Centre for Medical Imaging, University College London, 2nd floor Charles

Bell house, 43-45 Foley Street, London W1W 7TS, UK

2Department of Radiology, University College London Hospital, London, 235

Euston Road, London NW1 2BU, UK

Full list of author information is available at the end of the article
}

(c) The Author(s). 2019 Open Access This article is distributed under the terms of the Creative Commons Attribution 4.0 International License (http://creativecommons.org/licenses/by/4.0/), which permits unrestricted use, distribution, and reproduction in any medium, provided you give appropriate credit to the original author(s) and the source, provide a link to the Creative Commons license, and indicate if changes were made. The Creative Commons Public Domain Dedication waiver (http://creativecommons.org/publicdomain/zero/1.0/) applies to the data made available in this article, unless otherwise stated. 
(Continued from previous page)

Methods/design: The LOCATE trial is a prospective cohort, multi-centre, non-randomised, diagnostic accuracy study comparing WB-MRI and conventional imaging. Eligible patients will undergo WB-MRI in addition to conventional imaging investigations at the time of BCR and will be asked to attend a second WB-MRI exam, 12months following the initial scan. WB-MRI results will be compared to an enhanced reference standard comprising all the initial, follow-up imaging and non-imaging investigations. The diagnostic performance (sensitivity and specificity analysis) of WB-MRI for re-staging of BCR will be investigated against the enhanced reference standard on a per-patient basis. An economic analysis of WB-MRI compared to conventional imaging pathways will be performed to inform the cost-effectiveness of the WB-MRI imaging pathway. Additionally, an exploratory sub-study will be performed on blood samples and exosome-derived human epidermal growth factor receptor (HER) dimer measurements will be taken to investigate its significance in this cohort.

Discussion: The LOCATE trial will compare WB-MRI versus the conventional imaging pathway including its costeffectiveness, therefore informing the most accurate and efficient imaging pathway.

Trial registration: LOCATE trial was registered on ClinicalTrial.gov on 18th of October 2016 with registration reference number NCT02935816.

Keywords: Magnetic resonance imaging, Prostate cancer, Radiotherapy, Brachytherapy, Recurrence, Positron emission tomography, Cost comparison, Cost-effectiveness, Economic evaluation

\section{Background}

Prostate cancer $(\mathrm{PCa})$ is the leading male cancer in the United Kingdom and a principal cause of cancer-related mortality with around 46,700 cases diagnosed in 2014 [1]. The incidence of PCa has increased markedly over the past 20 years and it is projected to rise by $12 \%$ between 2014 and 2035 to 233 per 100,000 males [1]. Common treatments options for localised prostate cancer are external beam radiation therapy (EBRT), brachytherapy (BRT) or radical prostatectomy [2].

However, biochemical recurrence (BCR) following radiotherapy occurs in $25 \%$ of treated men within 5 years, manifesting as a rising prostate-specific-antigen (PSA) [3]. This is strictly a biochemical diagnosis, most commonly defined as an increase in serum PSA of 2.0 $\mathrm{ng} / \mathrm{mL}$ above the nadir [4]. Once a PSA relapse has been diagnosed, it is important to determine whether the recurrence has developed at local or distant sites and hence BCR following therapy often initiates an imagingbased assessment of local and metastatic disease in patients.

Local recurrence of $\mathrm{PCa}$ is investigated by prostate multi-parametric magnetic resonance imaging (mp-MRI) [5] whilst metastatic disease is assessed by multimodality imaging. The standard workup to detect PCa metastases usually includes ${ }^{99 \mathrm{~m}} \mathrm{Tc}$ bone scan and chest/ abdomen/pelvis (CT-CAP) scan (or where available ${ }^{18} \mathrm{~F}$-choline or ${ }^{68} \mathrm{Ga}$-PSMA PET-CT) [6].

The workup to exclude distant metastasis is of greatest significance as it is estimated that about half of those who develop radio-recurrent disease have distant metastases (either overt on imaging or micro-metastases) [7]. It is also believed that up to $50 \%$ of men who are considered to be free of metastases by conventional staging methods have micro-metastatic disease [8]. This is reflected in prostate cancer recurrence following EBRT or BRT, where biochemical relapse commonly precedes clinical detection of metastases by an average of 7-8 years [9].

Earlier detection of metastatic disease could be the key to better patient stratification and well-informed patient management either by opting for active surveillance or local salvage for low risk individuals or offering systemic therapy at an earlier time point to a high-risk population [10].

Recent technological advances have enabled the reliable whole-body MRI (WB-MRI) staging of cancers within a reasonable scanning time (less than $1 \mathrm{~h}$ ) [11-14]. There is a clear need for an imaging modality that is capable of improved sensitivity for metastatic disease detection than current conventional imaging [15-17]. Previous meta-analysis has reported the sensitivity of ${ }^{99 m}$ Tc bone scan to be less than $50 \%$ [18]. Similarly, studies have shown the sensitivity of ${ }^{18} \mathrm{~F}$-choline PET to be as low as $50 \%$ for the detection of nodal disease when PSA is $<5 \mathrm{ng} / \mathrm{ml}$ [19]. Furthermore, CT Scan sensitivity for nodal disease detection in prostate cancer is reported to be much lower with a meta-analysis reporting a sensitivity of $30 \%$ [20].

The LOCATE study investigates the diagnostic performance of WB-MRI for detection of nodal and metastatic disease compared to current standard multimodality imaging comprising ${ }^{18} \mathrm{~F}$-choline PET-CT and ${ }^{99 \mathrm{~m}} \mathrm{Tc}$ bone scan $(+/-\mathrm{CT}-\mathrm{CAP})$ in patients with the radio-recurrent $\mathrm{PCa}$. 


\section{Study objectives \\ Primary objective}

To compare the diagnostic accuracy of WB-MRI for regional lymph node and distant metastatic disease detection against an enhanced reference standard of ${ }^{18} \mathrm{~F}$ choline PET-CT (or CT CAP) and ${ }^{99 \mathrm{~m}} \mathrm{Tc}$ bone-scan for men presenting with $\mathrm{BCR}$ following EBRT or BRT.

\section{Secondary objectives}

1. Inter-observer agreement of WB-MRI for regional lymph node and distant metastatic disease detection

2. Derivation and evaluation of apparent diffusion coefficient (ADC) and fat fraction (FF) signal heterogeneity indices of metastatic disease as predictors of treatment response to ADT

3. Exploration of the significance of the Human Epidermal growth factor Receptor (HER) activated dimer in metastatic castration-resistant prostate cancer

4. Cost-effectiveness of WB-MRI for radio-recurrent prostate cancer staging compared with conventional imaging modalities

\section{Methods/design}

\section{Study design}

The comparative diagnostic accuracy study will conform to the Standards for Reporting of Diagnostic Accuracy (STARD) statement [21]. The study is a prospective cohort multi-centre diagnostic study where all participants will have WB-MRI and conventional imaging tests $\left({ }^{18} \mathrm{~F}\right.$ choline PET or CT-CAP and ${ }^{99 \mathrm{~m}} \mathrm{Tc}$ bone scan) with findings of both validated against an enhanced outcomebased reference standard. The patient recruitment flowchart is illustrated in Fig. 1.

\section{Sample size}

Based on $50 \%$ prevalence of metastases, 130 patients will be required to demonstrate a minimum of a $20 \%$ difference in sensitivity for detection of metastases on a perpatient level between WB-MRI and combination of ${ }^{18} \mathrm{~F}$ choline PET-CT and ${ }^{99 \mathrm{~m}} \mathrm{Tc}$ bone scan (power of $90 \%$ and statistical significance cut-off of 0.05 ).

\section{Ethical approval}

The LOCATE trial received UK Research Ethic Committee (REC) approval from the National research ethics service (NRES) Committee London-Chelsea with REC reference 15/LO/0776 and will be conducted in accordance with the principles of ICH guidelines on good clinical practice in clinical trials and the Research Governance Framework for Health and Social Care (England).

\section{Recruitment \\ Inclusion criteria}

Men who have undergone previous EBRT or BRT with or without neo-adjuvant/adjuvant hormone therapy.

Biochemical relapse as determined by increase in PSA.

\section{Exclusion criteria}

Men unable to have MRI scan, or in whom artefact would significantly reduce quality of MRI.

Men unable to give informed consent.

\section{Informed consent}

In all cases, patient information sheets and sample consent forms will be made available to patients a minimum of $24 \mathrm{~h}$ prior to the consent procedure. Consent will be obtained at the time of the appointment and prior to commencement of any trial intervention.

\section{Trial interventions Whole body MRI}

Recruited patients will have WB-MRI scan on presentation with BCR and then at 12 months (follow-up). All WB-MRI scans will be performed at University College London Hospital NHS foundation trust (UCLH) imaging department on a $3.0 \mathrm{~T}$ scanner (Ingenia; Phillips Healthcare, Best, Netherlands), using a fixed protocol (Table 1). For participants' comfort, the WB-MRI protocol will be limited to a maximum of 1-h scan time.

\section{Conventional imaging}

Recruited patients will have the standard imaging investigations according to local and national guidelines as below:

\section{Multi-parametric prostate MRI}

A standard prostate mp-MRI protocol as defined by the UK consensus guidelines on prostate MRI [22] will be used to locally stage prostate cancer. Conventional T1 and T2-weighted images of the prostate will be supplemented with diffusion weighted imaging (DWI) MRI (as per hospital site protocol); and $+/$ - dynamic contrast enhanced (DCE) T1-weighted images.

\section{Computed tomography}

CT scans of the chest/abdomen/pelvis will be performed with intravenous contrast agent using the local standard protocols.

\section{${ }^{99 m}$ Technetium bone scan}

Bone scans will be performed using ${ }^{99 \mathrm{~m}} \mathrm{Tc}$ labelled diphosphonates administered through intravenous injection. For prostate cancer patients with suspected bone metastases, the standard protocol employed at the hospital site will be used. As a guide, whole body imaging will be 


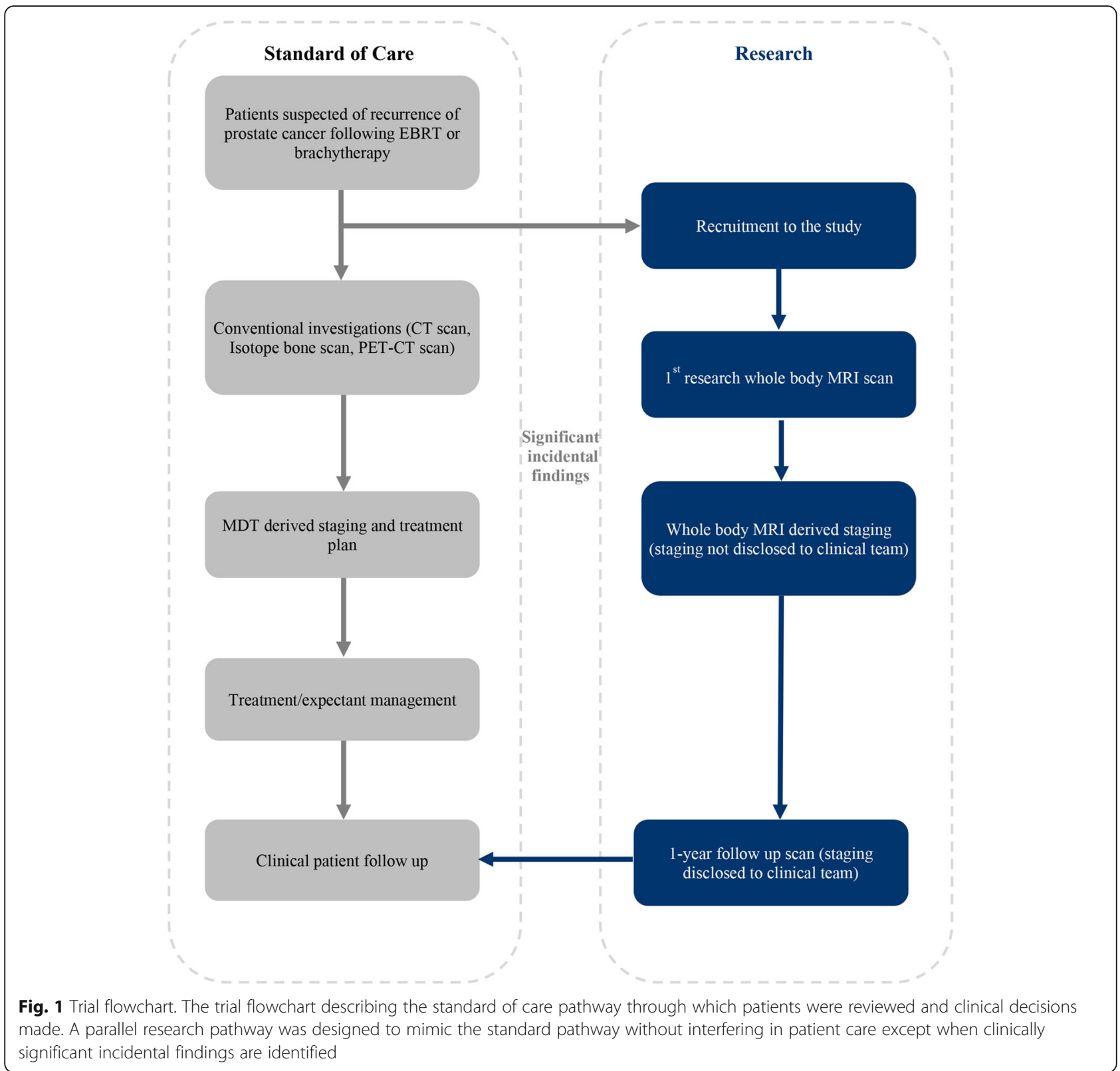

conventionally performed with anterior and posterior views, $256 \times 1024$ matrix and energy window(s) of 140 $\mathrm{KeV}$.

Positron emission tomography-computed tomography (PET-CT) ${ }^{18} \mathrm{~F}$-choline PET-CT will be acquired using a dedicated combined PET/64-detector-CT (VCT-XT Discovery, GEHealthcare Technology, Chicago, Illinois), CT will be performed (for attenuation correction) using $64 \times 3.75 \mathrm{~mm}$ detectors, a pitch of 1.5 and a $5 \mathrm{~mm}$ collimation $(120 \mathrm{kVp}$ and $10 \mathrm{~mA}$ in $0.8 \mathrm{~s}$ ). Maintaining the patient position, a whole-body choline PET emission scan will be performed and cover an area identical to that covered by CT [23].

\section{Non-imaging trial interventions} Blood samples

Blood samples will be taken from recruited patients, by an appropriately trained member of the trial team, at the time of each WB-MRI (staging and then at 12-month follow-up). Blood samples will be used for exosome and phenotypic [Fluorescence-activated cell sorting (FACS) analysis] / DNA-RNA analysis. In total a maximum of $30 \mathrm{ml}$ of blood will be taken:

Up to 3 acid citrate dextrose solution (ACD) tubes: to collect $\sim 15 \mathrm{ml}$ blood for serum separation

Up to 3 ethylenediaminetetraacetic acid (EDTA) containing tubes for peripheral blood mononuclear cells 
Table 1 Whole Body MRI sequence parameters

\begin{tabular}{llll}
\hline & T2-TSE & mDixon (pre- and post-contrast) & DWI (b0, 1000) \\
\hline Slice orientation & Transverse & Coronal & Transverse \\
Echo time (ms) & 80 & 2.303 & 17 \\
Repetition time (ms) & 1214.69 & 3.5 & 6304.5 \\
Space between slices & 5.5 & 2.5 & 5.5 \\
Number of slices & 40 & 120 & 40 \\
Slice thickness (mm) & 5 & 5 & 5 \\
Acquisition matrix & $500^{*} 497$ & $240^{*} 238$ & $124^{*} 118$ \\
Echo train length & 89 & 2 & 39 \\
Number of averages & 1 & 1 & 2 \\
Pixel bandwidth $(\mathrm{Hz})$ & 538 & 1847 & 3354 \\
Pixel spacing & $0.78 / 0.78$ & $1.04 / 1.04$ & 2.08 \\
Flip angle & 90 & 15 & 90 \\
\hline
\end{tabular}

TSE Turbo spin echo, DWI Diffusion weighted imaging

(PBMC) isolation and further FACS analysis, functional assays, and DNA/RNA isolation.

Frozen aliquots-samples will be transported by courier service in dry ice-containing carrier boxes within $24 \mathrm{~h}$ after obtaining the blood sample. Samples will be processed at University College London (UCL) and King's College London (KCL) laboratories:

UCL: Exosome purification and analysis. ProteinDNA-RNA isolation and analysis.

KCL: PBMC's isolation, FACS analysis and functional assays.

\section{Health economic data collection}

Data collection for the health economic analysis will be primarily concerned with cost data associated with the diagnosis and treatment of metastatic prostate cancer. Routinely collected administrative data from UCLH will be used to determine the relevant costs associated with the diagnostics test. To determine the consequence of detected metastatic disease on the treatment pathway, clinical experts will be interviewed, and patient notes will be reviewed noting changes to medication, radiotherapy, surgery, outpatient visits, inpatient visits and day cases, where possible. Data from existing trials such as STAMPEDE [24] and FORECAST [7] will be used to inform other economic parameters such as the health-related quality of life as these are not being collected in LOCATE.

\section{Reporting of trial imaging Initial WB-MRI scan}

The study will be reported by two experienced radiologists, blinded to all other imaging investigations, initially independently and then in consensus using a locked sequential read paradigm (LSR). The images will be reviewed for the presence of nodal $(\mathrm{N})$ and metastatic $(\mathrm{M})$ disease and each radiologist will record the presence/absence of disease on the study-specific proforma.

The initial WB-MRI read results will not be revealed to the clinical team. Where a significant additional/incidental finding is demonstrated this will be reported as per standard clinical practice and relayed to the clinical team.

A 1-6 Likert scale [23, 25] will be used for scoring as has been previously described for WB-MRI studies. 1 disease definitely not present, 2 - probably not present, 3 - possibly not present, 4 - disease possibly present, 5 - probably present and 6 - definitely present.

Scoring will be conducted based on evaluation of imaging features of lymph nodes and bone metastases as indicated below in Table 2 .

\section{2-month follow-up WB-MRI}

The study will be reported by the same pair of radiologists who reported the baseline scans. The findings on the follow-up study will be compared with the baseline WB-MRI with access to all conventional imaging. The presence of changes in size of lesions evident on the baseline WB-MRI and/or resolution of lesions evident on baseline WB-MRI will be recorded. Any discrepant findings will be reviewed, and the status of these lesions will be determined in a consensus panel review.

\section{Derivation of enhanced reference standard}

Figure 2 illustrates the derivation of a per-patient level enhanced reference standard (ERS).

Patients will be divided into those with and without metastases evident on conventional imaging. For those with metastases (left arm of Fig. 2), concordant positive WB-MRI findings will be deemed to be true positive and discordant WB-MRI findings will be considered to be false negative. 
Table 2 Features suggestive of adverse and benign changes in both nodes and bones

\begin{tabular}{|c|c|c|}
\hline & Benign features & Adverse features \\
\hline Node & $\begin{array}{l}\text { Lymph node }<5 \mathrm{~mm} \text { in short axis diameter (SAD) with no concerning } \\
\text { features on DWl, T2 weighted or contrast enhanced imaging and definite } \\
\text { benign features e.g. } \\
\text { (i) Fatty hilum, } \\
\text { (ii) Oval nodal morphology with clearly defined and } \\
\text { (iii) Regular nodal border and contours. }\end{array}$ & $\begin{array}{l}\text { Concerning features could be described as } \\
\text { (i) Size above normal limit e.g. > } 10 \text { mm for most, } \\
\text { (ii) Loss of fatty hilum, } \\
\text { (iii) Irregular border, } \\
\text { (iv) Asymmetric high DWI signal intensity, } \\
\text { (v) Low T2 signal }\end{array}$ \\
\hline Bone & $\begin{array}{l}\text { Normal appearing bone with uniform moderate-high signal on T1 } \\
\text { weighted imaging and low-moderate signal on T2 weighted imaging. } \\
\text { Focal lesions demonstrating clearly incidental/benign multiparametric } \\
\text { signal characteristics and/or location }\end{array}$ & $\begin{array}{l}\text { (i) Increase in DWI high b-value signal vs. background noise. } \\
\text { (ii) Low signal intensity on } \mathrm{T} 1 \text { and (iii) Intermediate to high signal } \\
\text { intensity on T2 } \\
\text { (iv) Lesional contrast enhancement }\end{array}$ \\
\hline
\end{tabular}

DWI Diffusion weighted imaging

For patients without metastases on conventional imaging (right arm of Fig. 2), clinical and biochemical follow-up together with changes between baseline and follow-up WB-MRI will be used to enhance the reference standard.

Where baseline WB-MRI was negative and concordant with conventional imaging, the presence of new lesions (at follow-up WB-MRI \pm repeat routine imaging) coupled with a PSA rise over the 12-month will classify the baseline WB-MRI as false negative, i.e. disease was present but not visualised. Whereas the absence of new lesions regardless of PSA status over the 12-month follow-up period will confer baseline WB-MRI true negative status.

For positive findings on baseline WB-MRI discordant with conventional imaging, the baseline WB-MRI will be deemed true positive when a new lesion declared itself (on follow-up WB-MRI \pm routine imaging) or the visualised lesion on baseline WB-MRI enlarged (on follow-up WB-MRI); and this was accompanied with a PSA rise within the 12-month follow-up period. Whereas, the

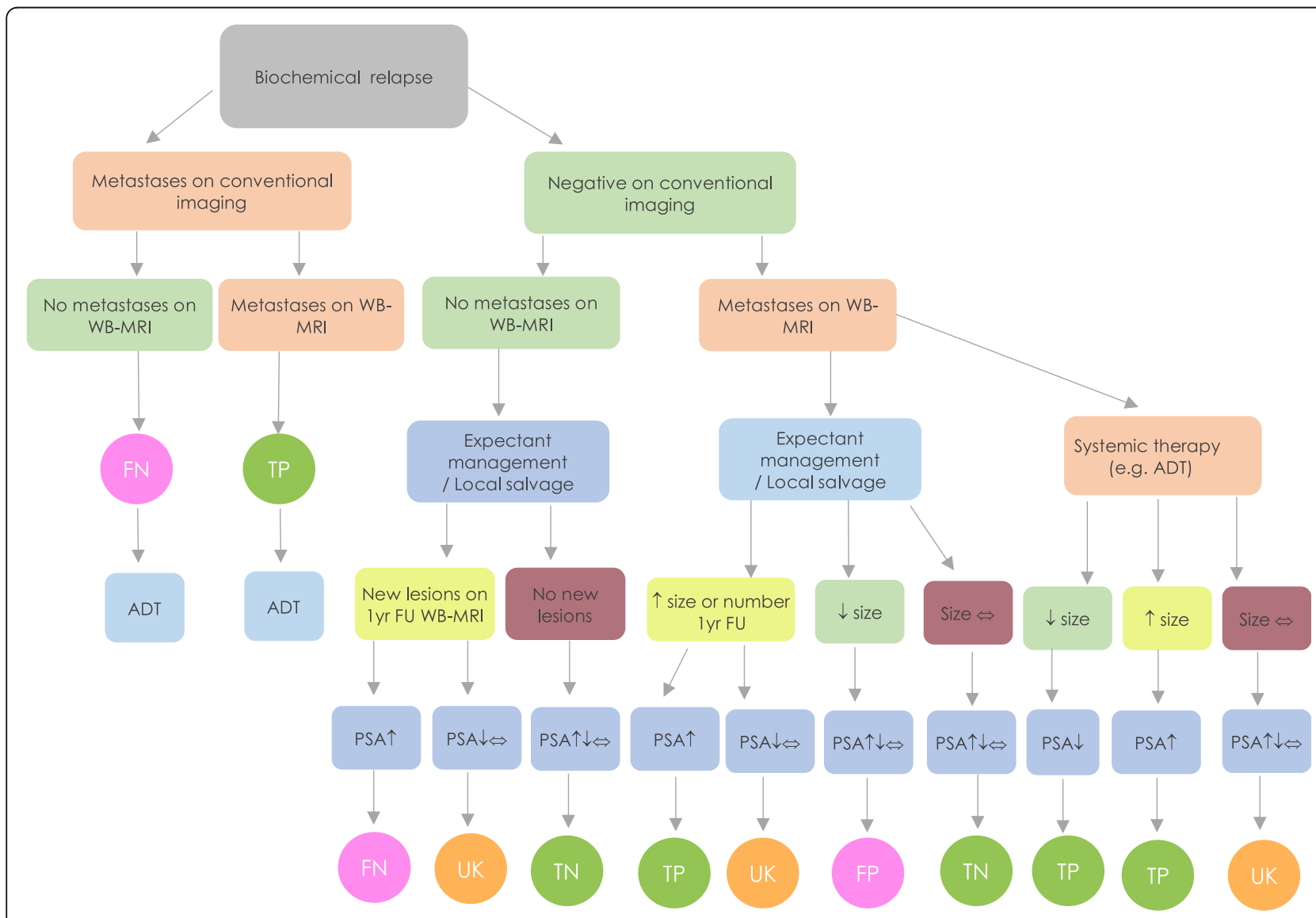

Fig. 2 Enhanced reference standard derivation flowchart. FN: False Negative, TP: True Positive, FP: False Positive, TN: True Negative, UK: Unknown, ADT: Androgen Deprivation Therapy 
baseline WB-MRI will be deemed false positive when the lesion evident at WB-MRI resolved or reduced in size at follow-up (in the absence of systemic therapy) regardless of PSA change. Lesions that resolved following systemic therapy (which is initiated in the 1-year followup period irrespective of negative conventional baseline imaging) with corresponding decrease in PSA will be classified as true positive.

Finally, patients with negative baseline conventional imaging with discordant lesion and PSA changes over the 12-month follow-up (i.e. lesion size/number increase without corresponding PSA increase) will be classified as unknown.

The section below describes the thresholds for PSA and lesion size changes, and they will be interpreted in the context of treatment intervention and subsequent treatment response or progression.

PSA progression defined as $\geq 25 \%$ and $\geq 2 \mathrm{ng} / \mathrm{mL}$ increase in PSA recorded at least 12 weeks from baseline or start of treatment and confirmed on a 2nd reading 3-4 weeks later as previously defined [26, 27].

PSA response defined as $\geq 50 \%$ decline from baseline or start of treatment and confirmed on a 2 nd reading 3-4 weeks later [28, 29].

Nodal size change Increase of $\geq 2 \mathrm{~mm}$ and decrease of $\geq 3 \mathrm{~mm}$ in short axis diameter on contrast enhanced mDixon sequence will be considered true change (progression and response respectively) rather than a measurement variation, with a $95 \%$ confidence interval. (Based on unpublished data from repeatability studies carried out as part of the LOCATE trial).

Bone lesion (disease progression) $\geq 25 \%$ increase in size (sum of short and long axes diameters) of lesions on CT, or MRI [30, 31].

Bone lesion (treatment response) $\geq 50 \%$ decrease in size (sum of short and long axes diameters) of lesions on CT/ MRI or normalisation of signal intensity on MRI [30, 31].

Soft tissue lesion size change (disease progression) At least a $20 \%$ increase in size of lesion taking as reference the baseline diameter of the lesion [32].

Soft tissue lesion size change (treatment response) at least a $30 \%$ decrease in the size of lesion, taking as reference the baseline diameter or disappearance of the lesion [32].

\section{Image analysis}

\section{Reporting of conventional imaging}

The conventional imaging $\left({ }^{18} \mathrm{~F}\right.$-choline PET-CT, CTCAP and ${ }^{99 \mathrm{~m}} \mathrm{Tc}$ Bone Scan) will be independently reported by two radiologists and nuclear medicine physicians, later reviewed in consensus to clarify any discrepant findings. The report will be transferred to a proforma and final nodal $(\mathrm{N})$ and metastatic $(\mathrm{M})$ stages will be derived base on the reported proforma.

\section{Comparison of conventional imaging with WB-MRI}

The research team will correlate the conventional imaging proforma with the baseline WB-MRI proforma and follow-up WB-MRI findings. The radiologists and nuclear medicine physicians in consensus will review all discrepancies, between conventional and WB-MRI scans. Anatomical matching errors resulting from discrepancies in ascribing disease location will be recorded and corrected [33]. Residual discrepancies will be highlighted, categorised as perceptual and technical and statistically assessed [33]. The per-patient sensitivity and specificity of WB-MRI will be determined against the enhanced reference standard before and after correction for any anatomical matching discrepancies and perceptual errors.

Patients who are unable to attend the 1 year follow up WB-MRI may not be included in the diagnostic accuracy analysis. Efforts will be made to minimise amount of missing data. A statistician's advice will be sought especially on how to manage 'missing not at random' data (if any).

\section{Sub-study analysis \\ The significance of the human epidermal growth factor receptor (HER) activated dimer}

The blood sample will be used to investigate the significance of the Human Epidermal growth factor Receptor (HER) activated dimer in recurrent prostate cancer. Upregulation of the HER1 (EGFR)/HER3 dimer was recently found, for the first time, to limit the efficacy of anti- epidermal growth factor receptor (EGFR) treatment in human breast cancer, as directly shown by imaging of residual disease; suggesting the potential of combined EGFR/HER3-targeted treatment [34]. This molecular signalling rewiring is hypothesised to constitute a pathway of resistance to hormonal treatment in prostate cancer as well. We intend to quantify the EGFR/HER3 heterodimer using fluorescence lifetime imaging microscopy (FLIM) in formalin-fixed prostate cancer tissues as well as in matched circulating exosomes from patientderived plasma. We will then correlate the heterodimer quantification with tumour genomic changes such as phosphatase and tensin homolog (PTEN) mutation/deletion. The patient-derived correlative experiments will be complemented by mechanistic in vitro experiments that investigate the effect of EGFR and phosphatidylinositol 3-kinase (PI3K)/AKT inhibitors on HER dimer formation in castration-resistant and sensitive prostate cancer cells, and their exosomes released into the culture supernatants. In the clinical setting, the tissue and exosomederived HER dimer measurements will be combined with WB-MRI findings to assess their value and 
translation as predictive biomarkers of clinical outcome and response to treatment.

\section{Heterogeneity (across metastatic sites) of WB-MRI signals for prediction of ADT response}

For patients undergoing ADT treatment, retrospective quantitative analysis of WB-MRI's ADC and FF signal intensities will be performed to derive heterogeneity indices across confirmed true positive metastatic sites. The heterogeneity indices will inform Bayesian models to predict high-risk patients with aggressive disease that progress on ADT within 12-months of treatment.

\section{Health economic analysis}

The health economic analysis will compare the incremental costs and effects of WB-MRI for metastatic disease detection compared with conventional imaging from a national health service (NHS) perspective and thus determine whether it is an efficient use of NHS resources. From an economic viewpoint, the costeffectiveness of WB-MRI versus conventional imaging is determined by two main stages of the pathway: the treatment decision pathway and the treatment pathway (and its associated disease pathway). The former includes the time from presentation to treatment decision by the clinician and includes the imaging tests received; the latter includes the time period following the treatment decision and subsequent disease progression. In patients for whom the treatment decision with WB-MRI is the same as that with conventional staging, the subsequent disease pathways will be the same. Where the treatment decision with WB-MRI is different, the disease pathway will be different, yielding potentially different costs and health outcomes (and hence the patient's quality of life).

The precise nature of the economic analysis will therefore depend on the degree of concordance between treatment decisions provoked by WB-MRI versus conventional imaging.

\section{Scenario 1}

There is concordance between the treatment decisions associated with WB-MRI and conventional imaging. In this case, the cost components included in the analysis will be conventional metastases imaging tests (as described above) and the costs of treating adverse events associated with conventional staging. The volume of resource use and unit costs will be taken from standard published sources, hospital administrative datasets and from trial data. Since the two algorithms yield the same treatment decisions, cost-effectiveness depends on the incremental cost only of WB-MRI versus conventional imaging.

\section{Scenario 2}

There is discordance between the treatment decisions associated with WB-MRI and standard imaging. In this case, cost-effectiveness depends on the incremental cost and effects of the treatment decision pathway and the subsequent disease pathway associated with WB-MRI versus conventional staging.

The economic evaluation will also take into consideration the accuracy of WB-MRI versus conventional standard imaging techniques. Specifically, we will model the consequences, in both costs and effects, of true positives, false positives, true negatives and false negative outcomes. Thus, the economic study will evaluate both clinical and economic consequences of the novel imaging technique.

Cost-effectiveness will be calculated as the mean cost difference between WB-MRI versus conventional imaging divided by the mean difference in outcomes (as measured quality-adjusted life years; QALYs) to give the incremental cost-effectiveness ratio (ICER). Also, a costconsequence analysis will be performed by calculating the mean incremental costs per incremental gain (or loss) in clinical natural units, for example cost per metastases identified.

Finally, to address uncertainty in our clinical and economic parameters, we will perform a comprehensive set of sensitivity analyses on our model estimates. Probabilistic sensitivity analysis using non-parametric bootstrapping methods will be performed. Outputs from this will inform a cost-effectiveness acceptability curve, which shows the probability that WB-MRI is cost-effective at different values of the NHS' willingness to pay for an additional QALY. We will also subject the economic model to extensive one-way sensitivity analysis to identify key parameters that drive cost-effectiveness.

\section{Discussion}

At the time of writing, the LOCATE trial is the largest prospective multi-centre trial to compare WB-MRI with conventional imaging pathway specifically in patients with radio-recurrent prostate cancer.

The LOCATE trial will be informative because it compares comprehensive imaging pathways and takes into consideration the cost-effectiveness of each pathway. This trial will therefore provide valuable information to guide potential implementation of new imaging platforms for prostate cancer management pathways.

The results from the LOCATE trial would be relevant to the current clinical management pathway, specifically, should WB-MRI be advocated in the NHS in the setting of BCR.

The LOCATE trial design includes important features: unlike most other trials, the initial finding on WB-MRI will be validated using 1 year follow up WB-MRI scans, 
corroborating the findings on initial scan in the context of patient management. In this way, we minimise the potential overcalling of disease positivity by WB-MRI that could lead to an overestimated sensitivity of WB-MRI compared to conventional imaging techniques.

Additionally, and in order to define the most costeffective imaging pathway for the health care system, we will perform an economic evaluation which includes a comprehensive set of sensitivity analyses to identify uncertainty in both clinical and economic estimates. Whilst recent trial designs incorporating WB-MRI are including health economic analysis [35-38], such information is still unavailable for prostate cancer imaging pathways.

The LOCATE study has some potential limitations. Firstly, we are using a combined biochemical/imaging follow-up to derive an enhanced reference standard against which the results of WB-MRI will be analysed. Although histopathological confirmation would have been preferable in cases where a discrepancy exists between WB-MRI and conventional imaging tests, such approach is technically challenging and ethically not feasible [39].

Secondly, whilst the qualitative and metastatic staging findings can be generalised to other imaging platforms, such generalisability would be limited in quantitative biomarker imaging analyses (heterogeneity index). However, ongoing work is being carried out to improve standardisation of quantitative MRI imaging across different platforms that could ultimately aid in generalisation of such technique [39, 40].

Finally, it is important to note that ${ }^{68} \mathrm{Ga}$-PSMA PET-CT within a short time has led to significant advances in prostate cancer imaging both in primary and recurrent settings [41, 42]. However, there is yet to be large prospective multi-centre studies to assess its diagnostic accuracy in comparison to WB-MRI, and the start of the LOCATE trial predated availability of ${ }^{68} \mathrm{Ga}$ PSMA PET-CT at our institution and therefore a head to head comparison was not possible. More importantly, there is significant cost difference between the two modalities and PSMA is routinely not available in many centres in the UK.

\section{Study status}

At the time of submission, the study is currently at the data collection stage and full trial data analysis is expected to begin shortly.

\section{Abbreviations}

ACD: Acid citrate dextrose; ADC: Apparent diffusion coefficient; ADT: Androgen deprivation therapy; BCR: Biochemical relapse; BRT: Brachytherapy; CT: Computed tomography; DWI: Diffusion weighted imaging, EBRT: External beam radiotherapy;

EDTA: Ethylenediaminetetraacetic acid; EFGR: Epidermal growth factor receptor; EPI: Echo planar imaging; FACS: Fluorescence-activated cell sorting; FF: Fat fraction; FLIM: Fluorescence lifetime imaging microscopy; HER: Human epidermal growth factor receptor; ICER: Incremental cost-effectiveness ratio; MDT: Multi-disciplinary team; MRI: Magnetic resonance imaging; NHS: National health service; PACS: picture and archiving system; PBMC: Peripheral blood mononuclear cell; PCa: Prostate cancer; PET: Positron emission tomography; PI3K/AKT: Phosphatidylinositol 3-kinase; PSA: Prostate specific antigen; QALY: Quality-adjusted life year; TSE: Turbo spin echo; WBMRI: Whole-body magnetic resonance imaging

\section{Acknowledgments}

We would like to thank all the patients who have volunteered for this study, the site teams and principal Investigators who have worked on this study and made it possible.

\section{Authors' contributions}

Study concept and initial design: SP, TN, AL, HUA, SM, GC. Study design and statistical analysis: SP, TN, SA, AL, HUA, SM, AC. Health economic analysis SV,SM, SA. Acquisition of data and data analysis and interpretation: SP, SA, AL, TS, SM, SV, MG, TN, HS, RD, HAP, JC, MA, AH, SW, SC, AC, GC, HUA, MC, $\mathrm{ML}, \mathrm{SUC}, \mathrm{AF}, \mathrm{MRJ}$. All authors read and approved the final manuscript.

\section{Funding}

This project was funded by Prostate Cancer UK (PCUK), project grant reference PG13-026. The work of Sola Adeleke is supported by Cancer Research UK \& Engineering and physical sciences research grant (EPSRC) comprehensive cancer imaging centre at UCL (CCIC) project number C1519/ A16463. The work of Arash Latifoltojar is supported by Prostate Cancer UK (PCUK) grant, project number PG13-026. The work of Shonit Punwani is supported by the UCL/UCLH Biomedical Research Centre.

Hashim Ahmed reports receipt of grant funding and personal fees from SonaCare Medical Inc., grant funding from Trod Medical, and grant funding and personal fees from Sophiris Bio Inc. Ahmed also receives grant funding from Wellcome Trust, MRC (UK), UK NIHR Imperial Biomedical Research Centre, Prostate Cancer UK, The Urology Foundation and Imperial Healthcare Charity. Ahmed receives support from United Kingdom's NIHR Imperial Biomedical Research Centre.

The work of Tony $\mathrm{Ng}$ and Myria Galazi are in part supported by Cancer Research-UK (grants C1519/A6906 and C5255/A15935); the King's College London-UCL Comprehensive Cancer Imaging Centre (CRUK \& EPSRC) and in association with the Medical Research Council (MRC) and Department of Health (DoH) (grants C1519/ A16463 and C1519/A1033.

The funders had no role in the study design, data collection and analysis, decision to publish and preparation of the manuscript. Department of Health Disclaimer: The views and opinions expressed therein are those of the authors and do not necessarily reflect those of Prostate Cancer UK, the NHS or the Department of Health.

\section{Availability of data and materials}

Not applicable.

\section{Ethics approval and consent to participate}

The study abides by the principles of the Declaration of Helsinki and the UK Research Governance Framework version 2.

LOCATE trial received UK Research Ethic Committee approval on 19th of June 2015 by the NRES Committee London-Chelsea with REC reference 15/ $\mathrm{LO} / 0776$ and is registered on clinicaltrials.gov with reference number NCT02935816.

Written informed consent were obtained from all study participants. Findings from this research will be disseminated at conferences and submitted for peer-reviewed publications.

\section{Consent for publication}

Not applicable.

\section{Competing interests}

The study has not received any funding/assistance from a commercial organisation. The authors declare that they have no competing interests.

\section{Author details}

${ }^{1}$ Centre for Medical Imaging, University College London, 2nd floor Charles Bell house, 43-45 Foley Street, London W1W 7TS, UK. ²Department of Radiology, University College London Hospital, London, 235 Euston Road, 
London NW1 2BU, UK. ${ }^{3}$ Molecular Oncology Group, University College London, Cancer Institute, Paul O'Gorman Building, 72 Huntley Street, London WC1E 6DD, UK. ${ }^{4}$ Division of Surgery and Interventional Science, University College London, 4th floor, 21 University Street, London WC1E, UK. ${ }^{5}$ Division of Surgery, Department of Surgery and Cancer, Faculty of Medicine, Imperial College London, London, UK. ${ }^{6}$ Department of Urology, Charing Cross Hospital, Imperial College Healthcare NHS Trust, London, UK. 'Oncology Department, University College London Hospital, 235 Euston Road, London NW1 2BU, UK. ${ }^{8}$ Comprehensive Cancer Imaging Centre (CCIC), King's College, London, New Hunt's House, Guy's Campus, London SE1 1UL, UK. ${ }^{9}$ Department of Nuclear Medicine, The Royal Marsden Hospital NHS Foundation Trust, Down's Road, Sutton SM2 5PT, UK. ${ }^{10}$ Histopathology Department, University College London Hospital, 4th Floor, Rockefeller Building University Street, London WC1 6DE, UK. " II Institute for Mathematical and Molecular Biomedicine, King's College London, Hodgkin Building, Guy's Campus, London SE1 1UL, UK. ${ }^{12}$ Department of Applied Health Research, University College London, 1-19 Torrington Place, Fitzrovia, London WC1E 7HB, UK. ${ }^{13}$ Department of Cancer Imaging, School of Biomedical Engineering and Imaging Sciences, King's College London, 4th Floor, Lambeth Wing St. Thomas' Hospital, London SE1 7EH, UK. ${ }^{14}$ Institute of Nuclear Medicine, University College London Hospital, 5th Floor Tower, 235 Euston Road, London NW1 2BU, UK. ${ }^{15}$ Urology Department, University College Hospital, Westmoreland Street, 16-18 Westmoreland Street, London W1G 8PH, UK. ${ }^{16}$ Oncology Department, Guy's and St. Thomas' Hospital, Westminster Bridge road, Lambeth, London SE1 7EH, UK. ${ }^{17}$ Department of Nuclear Medicine, St Bartholomew's Hospital, West Smithfield, London EC1A 7BE, UK. ${ }^{18}$ Urology Department, Imperial College Healthcare NHS Trust, London W2 1NY, UK.

Received: 23 July 2019 Accepted: 13 September 2019

Published online: 15 November 2019

\section{References}

1. http://www.cancerresearchuk.org/health-professional/cancer-statistics/ statistics-by-cancer-type/prostate-cancer\#heading-Zero- Accessed 18th of June 2019.

2. Naik M, Marta GN, Abdel-Wahab M. Reirradiation of locally recurrent prostate cancer after primary radiotherapy. J Radiat Oncol. 2015;4(2):149-56.

3. Shilkrut M, McLaughlin PW, Merrick GS, Vainshtein JM, Feng FY, Hamstra DA. Interval to biochemical failure predicts clinical outcomes in patients with high-risk prostate cancer treated by combined-modality radiation therapy. Int J Radiat Oncol Biol Phys. 2013;86(4):721-8.

4. Roach M 3rd, Hanks G, Thames H Jr, Schellhammer P, Shipley WU, Sokol GH, Sandler $\mathrm{H}$. Defining biochemical failure following radiotherapy with or without hormonal therapy in men with clinically localized prostate cancer: recommendations of the RTOG-ASTRO Phoenix consensus conference. Int J Radiat Oncol Biol Phys. 2006;65(4):965-74.

5. Turkbey B, Brown AM, Sankineni S, Wood BJ, Pinto PA, Choyke PL. Multiparametric prostate magnetic resonance imaging in the evaluation of prostate Cancer. CA Cancer J Clin. 2016;66(4):326-36.

6. Testa C, Pultrone C, Manners DN, Schiavina R, Lodi R. Metabolic imaging in prostate Cancer: where we are. Front Oncol. 2016;6:225.

7. Kanthabalan A, Shah T, Arya M, Punwani S, Bomanji J, Haroon A, Illing RO, Latifoltojar A, Freeman A, Jameson C, van der Meulen J, Charman S, Emberton M, Ahmed HU. The FORECAST study — focal recurrent assessment and salvage treatment for radiorecurrent prostate cancer. Contemp Clin Trials. 2015:44:175-86.

8. Edelstein RA, Zietman AL, De Las MA, Krane RJ, Babayan RK, Dallow KC, Moreland RB. Implications of prostate micro-metastases in pelvic lymph nodes: an archival tissue study. Urology. 1996;47(3):370-5.

9. Schiavina R, Ceci F, Borghesi M, Brunocilla E, Vagnoni V, Gacci M, Castellucci P, Nanni C, Martorana G, Fanti S. The dilemma of localizing disease relapse after radical treatment for prostate Cancer: which is the value of the actual imaging techniques? Curr Radiopharm. 2013;6(2):92-5.

10. Gillessen S, Atard G, Beer TM, Beltran H, Bossi A, Bristow R, et al. Management of patients with advanced prostate cancer: the report of the advanced prostate cancer consensus conference APCCC 2017. Eur Urol. 2018;73(2):178-211.

11. Eiber M, Holzapfel K, Ganter C, Epple K, Metz S, Geinitz H, Kubler H, Gaa J, Rummeny EJ, Beer AJ. Whole-body MRI including diffusion-weighted imaging (DWI) for patients with recurring prostate cancer: technical feasibility and assessment of lesion conspicuity in DWI. J Magn Reson Imaging. 2011;33(5):1160-70.

12. Pasoglou V, Larbi A, Collette L, Annet L, Jamar F, Machiels JP, Michoux N, Vande Berg BC, Tombal B, Lecouvet FE. One-step TNM staging of high-risk prostate cancer using magnetic resonance imaging (MRI): toward an upfront simplified "all-in-one" imaging approach? Prostate. 2014;74(5):496-77.

13. Lecouvet FE, El Mouedden J, Collette L, Coche E, Danse E, Jamar F, Machiels JB, Vande Berg B, Omoumi P, Tombal B. Can whole-body magnetic resonance imaging with diffusion-weighted imaging replace Tc-99m bone scanning and computed tomography for single-step detection of metastases in patients with high-risk prostate cancer? Eur Urol. 2012;62(1):68-75.

14. Robertson NL, Sala E, Benz M, Landa J, Scardino P, Scher HI, Hricak H, Vargas HA. Combined whole body and multiparametric prostate magnetic resonance imaging as a 1-step approach to the simultaneous assessment of local recurrence and metastatic disease after radical prostatectomy. J Urol. 2017;198(1):65-70

15. Briganti A, Abdollah F, Nini A, Suardi N, Gallina A, Capitanio U, Bianchi M, Tutolo M, Passoni NM, Salonia A, Colombo R, Freschi M, Rigatti P, Montorsi F. Performance characteristics of computed tomography in detecting lymph node metastases in contemporary patients with prostate cancer treated with extended pelvic lymph node dissection. Eur Urol. 2012;61(6):1132-8.

16. Talab SS, Preston MA, Elmi A, Tabatabaei S. Prostate cancer imaging: what the urologist wants to know. Radiol Clin N Am. 2012;50(6):1015-41.

17. Sartor O, Eisenberger M, Kattan MW, Tombal B, Lecouvet F. Unmet needs in the prediction and detection of metastases in prostate cancer. Oncologist. 2013;18(5):549-57.

18. Suh $\mathrm{CH}$, Shinagare $\mathrm{AB}$, Westenfield $\mathrm{AM}$, Ramaiya $\mathrm{NH}$, Van den Abbeele $\mathrm{AB}$, Kim KW. Yield of bone scintigraphy for the detection of metastatic disease in treatment-naïve prostate cancer: a systemic review and meta-analysis. Clin Radiol. 2018;73(2):158-67.

19. Rinnab L, Mottaghy FM, Simon J, Volkmer BG, De Petriconi R, Hautman RE, et al. ${ }^{11} \mathrm{C}$ choline PET/CT for targeted salvage lymph node dissection in patients with biochemical recurrence after primary curative therapy for prostate cancer: preliminary results of a prospective study. Urol Int. 2008;81:191-7.

20. Abuzallouf S, Dayes I, Lukka H. Baseline staging of newly diagnosed prostate cancer: a summary of the literature. J Urol. 2004;171:2122-7.

21. Bossuyt PM, Reitsma JB, Bruns DE, Gatsonis CA, Glasziou PP, Irwig L, Lijmer JG, Moher D, Rennie D, de Vet HC, Kressel HY, Rifai N, Golub RM, Altman DG, Hooft L, Korevaar DA, Cohen JF. STARD group. STARD 2015: an updated list of essential items for reporting diagnostic accuracy studies. Radiology. 2015;277(3):826-32

22. Kirkham APS, Haslam P, Keanie JY, McCafferty I, Padhani AR, Punwani S, Richenberg J, Rottenberg G, Sohaib A, Thompson P, Turnbull LW, Sahdev A, Clements R, Carey BM, Allen C. Prostate MRI: Who, when, and how? Report from a UK consensus meeting. Clin Radiol. 2013;68(10):1016-23.

23. Johnston EW, Latifoltojar A, Sidhu HS, Ramachandran N, Sokolska M, Bainbridge A, et al. Multiparametric whole-body 3.0-T MRI in newly diagnosed intermediate- and high-risk prostate cancer: diagnostic accuracy and interobserver agreement for nodal and metastatic staging. Eur Radiol. 2019;29(6):3159-69.

24. Carthon BC, Antonarakis ES. The STAMPEDE trial: paradigm-changing data through innovative trial design. Transl Cancer Res. 2016;5(S3):S485-90.

25. Shin T, Smyth TB, Ukimura O, Ahmadi N, de Castro Abreu AL, Ohe C, et al. Diagnostic accuracy of a five-point Likert scoring system for magnetic resonance imaging (MRI) evaluated according to results of MRI/ ultrasonography image-fusion targeted biopsy of the prostate. BJU Int. 2018; 121(1):77-83.

26. Scher HI, Halabi S, Tannock I, Morris M, Sternberg CN, Carducci MA, et al. Design and end points of clinical trials for patients with progressive prostate cancer and castrate levels of testosterone: recommendations of the prostate Cancer clinical trials working group. J Clin Oncol. 2008;26(7):1148-59.

27. Scher HI, Morris MJ, Stadler WM, Higano C, Basch E, Fizazi K, et al. Trial design and objectives for castration-resistant prostate cancer: updated recommendations from the prostate cancer clinical trials working group 3. J Clin Oncol. 2016:34(12):1402-18.

28. de Bono JS, Logothetis CJ, Molina A, Fizazi K, North S, Chu L, et al. Abiraterone and increased survival in metastatic prostate cancer. N Engl J Med. 2011;364(21):1995-2005.

29. Value of PSA as a Tumour Marker. Available from: https://oncologypro.esmo. org/content/download/98717/1732452/file/ESMO-Preceptroship-prostatecancer-lugano-november-2016-CASTRO.pdf . 
30. Costelloe CM, Chuang HH, Madewell JE, Ueno NT. Cancer response criteria and bone metastases: RECIST 1.1, MDA and PERCIST. J Cancer. 2010;1:80-92.

31. Hamaoka T, Madewell JE, Podoloff DA, Hortobagyi GN, Ueno NT. Bone imaging in metastatic breast cancer. J Clin Oncol. 2004;22(14):2942-53.

32. Eisenhauer EA, Therasse P, Bogaerts J, Schwartz LH, Sargent D, Ford R, et al. New response evaluation criteria in solid tumours: revised RECIST guideline (version 1.1). Eur J Cancer. 2009;45(2):288-47.

33. Latifoltojar A, Punwani S, Lopes A, Humphries PD, Klusmann M, Menezes LJ, Daw S, Shankar A, Neriman D, Fitzke H, Clifton-Hadley L, Smith P, Taylor SA. Whole-body MRI for staging and interim response monitoring in paediatric and adolescent Hodgkin's lymphoma: a comparison with multi-modality reference standard including 18F-FDG-PET-CT. Eur Radiol. 2019;29(1):202-12.

34. Nuciforo P, Radosevic-Robin N, Ng T, Scaltriti M. Quantification of HER family receptors in breast cancer. Breast Cancer Res. 2015;17:53.

35. Taylor SA, Mallett S, Miles A, Beare S, Bhatnagar G, Bridgewater J, GlynneJones R, Goh V, Groves AM, Janes SM, Koh DM, Morris S, Morton A, Navani N, Oliver A, Padhani AR, Punwani S, Rockall AG, Halligan S. Streamlining staging of lung and colorectal cancer with whole body MRl; study protocols for two multicentre, non-randomised, single-arm, prospective diagnostic accuracy studies (Streamline C and Streamline L). BMC Cancer. 2017 May;2; 17(1):299.

36. Taylor SA, Mallett S, Beare S, et al. Diagnostic accuracy of whole-body MRI versus standard imaging pathways for metastatic disease in newly diagnosed colorectal cancer: the prospective streamline C trial. Lancet Gastroenterol Hepatol. 2019; Epub ahead of print.

37. Taylor SA, Mallett S, Ball S, et al. Diagnostic accuracy of whole-body MRI versus standard imaging pathways for metastatic disease in newly diagnosed non-small-cell lung cancer: the prospective streamline $L$ trial. Lancet Resp. 2019; Epub ahead of print.

38. Johnston E, Pye H, Bonet-Carne E, Panagiotaki E, Patel D, Galazi M, Heavey S, Carmona L, Freeman A, Trevisan G, Allen C, Kirkham A, Burling K, Stevens N, Hawkes D, Emberton M, Moore C, Ahmed HU, Atkinson D, RodriguezJusto M, Ng T, Alexander D, Whitaker H, Punwani S. INNOVATE: a prospective cohort study combining serum and urinary biomarkers with novel diffusion-weighted magnetic resonance imaging for the prediction and characterization of prostate cancer. BMC Cancer. 2016;16(1):816.

39. Barnes A, Alonzi R, Blackledge M, Charles-Edwards G, Collins DJ, Cook G, Coutts G, Goh V, Graves M, Kelly C, Koh DM, McCallum H, Miquel ME, O'Connor J, Padhani A, Pearson R, Priest A, Rockall A, Stirling J, Taylor S, Tunariu N, van der Meulen J, Walls D, Winfield J, Punwani S. UK quantitative WB-DWI technical workgroup: consensus meeting recommendations on optimisation, quality control, processing and analysis of quantitative wholebody diffusion-weighted imaging for cancer. Br J Radiol. 2018 Jan;91(1081): 20170577.

40. Padhani AR, Lecouvet FE, Tunariu N, Koh DM, De Keyzer F, Collins DJ, Sala E, Schlemmer HP, Petralia G, Vargas HA, Fanti S, Tombal HB, de Bono J. METastasis reporting and data system for prostate Cancer: practical guidelines for acquisition, interpretation, and reporting of whole-body magnetic resonance imaging-based evaluations of multiorgan involvement in advanced prostate Cancer. Eur Urol. 2017;71(1):81-92.

41. Kallur K, Ramachandra P, Rajkumar K, Swamy S, Desai I, Rao R, et al. Clinical utility of gallium-68 PSMA PET/CT scan for prostate cancer. Indian J Nucl Med. 2017;32(2):110.

42. Perera M, Papa N, Christidis D, Wetherell D, Hofman MS, Murphy DG, et al. Sensitivity, specificity, and predictors of positive 68Ga-prostate-specific membrane antigen positron emission tomography in advanced prostate Cancer: a systematic review and meta-analysis. Eur Urol. 2016;70(6):926-37.

\section{Publisher's Note}

Springer Nature remains neutral with regard to jurisdictional claims in published maps and institutional affiliations.

Ready to submit your research? Choose BMC and benefit from:

- fast, convenient online submission

- thorough peer review by experienced researchers in your field

- rapid publication on acceptance

- support for research data, including large and complex data types

- gold Open Access which fosters wider collaboration and increased citations

- maximum visibility for your research: over $100 \mathrm{M}$ website views per year

At BMC, research is always in progress.

Learn more biomedcentral.com/submissions 